\title{
A comparison between the sensitivities of VLC receivers containing an off-the-shelf SPAD array and an APD
}

Long Zhang, Hyunchae Chun, Grahame Faulkner, Dominic O’Brien and Steve Collins

Department of Engineering Science University of Oxford, Oxford, UK. (steve.collins@eng.ox.ac.uk)

Abstract This paper proposes a method to detect photon counts from a commercial off-the-shelf SPAD array.
Experiments show that using this method the sensitivity of a VLC receiver that incorporates this SPAD array approaches the limit set by Poisson noise.

\section{Introduction}

Visible light communications (VLC) has been proposed as a method of providing indoor communications without the problems that can occur when using radio-frequency (RF) communications[1]. In a VLC system, data is encoded by varying the transmitted optical power and hence the sensitivity of a receiver has a significant impact on the performance of any VLC system. Recently, single photon avalanche detectors (SPADs) have become available that are capable of detecting individual photons $[2,3]$. This means that ideally the sensitivity of a SPAD based VLC receiver will be determined by Poisson noise and they will be more sensitive than any existing receivers. However, SPADs are still a relatively immature technology whose sensitivity is reduced by relatively low photon detection efficiencies and the unavoidable dead-time after a photon is detected. Results with small arrays of SPADs show that the negative impact of dead-time can be mitigated by using arrays of SPADs whose dead-time is shorter than the time between photons crossing the active area of each $\operatorname{SPAD}[4,5,6,7]$. However, these results were obtained using prototype SPAD arrays that are not widely available.

In this paper, we describe two simple methods of counting the photons detected by a commercial offthe-shelf array of SPADs. The performance of a VLC receiver that uses this array and the best of these two methods of counting photons is then compared to the performance of both an APD-based receiver and a system limited by Poisson noise.

\section{Photon counting method}

The commercial off-the-shelf detector that was used for our experiments is the C11209-110 optical measurement module. This module includes an S12571-010C photodetector containing an array of $10^{4}$ SPADs with a diameter of $10 \mu \mathrm{m}$ in an area of $1 \mathrm{~mm}$ by $1 \mathrm{~mm}$ that share a common output. As shown in Fig. 1 10ns wide pulses of varying heights are observed when photons are detected, however, a histogram of these pulse heights shows that they are all approximately integer values of $4 \mathrm{mV}$. The two smaller pulses in Fig. 1 are therefore caused by single photons whilst the larger pulse is caused by two photons that are detected simultaneously.

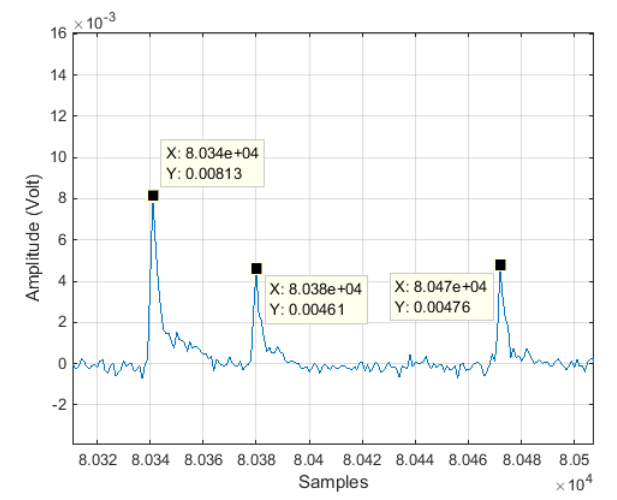

Fig. 1: The typical output waveform from the C11209-110.

The pulse heights mean that photons can be counted by finding the peak of each pulse, dividing this value by a threshold value $(3.78 \mathrm{mV})$ and rounding the result to the nearest integer value. The number of detected photons in a bit period is then the sum of these integers in a bit period. Although this method is simple there is a possibility that the peak from a photon might be obscured by the tail of a large peak caused by several photons being detected simultaneously. In order to avoid problems that this might cause photons have also been counted by summing the output voltages observed within a specific period and dividing this value by a threshold value $(22 \mathrm{mV})$ that represents the sum obtained from a typical response to a single photon.

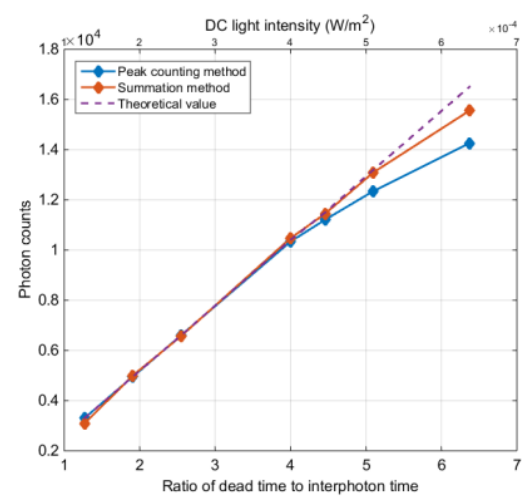

Fig. 2: The counts of detected photons within $100 \mu \mathrm{s}$ at different light levels. In this figure the dashed line shows the expected number of detected photons in this fixed time interval. The solid lines then show the number of detected photons in the same period determined by the two photon counting methods. 
For any photon-counting method, the dead time and inter-photon time are the two critical times. The results in Fig. 2 are therefore presented as a function of both the light intensity falling on the SPAD array and the ratio of these two critical times. The results in Fig. 2 show that the summation method is slightly more accurate than the peak counting method. In particular, it works perfectly when there are fewer than 5 detected photons within 10 ns.

\section{MPPC based VLC demonstration}

To determine the sensitivity of the C11209-110 when it acts as a receiver it has been incorporated into a communications link. The transmitter for this link was a $650 \mathrm{~nm}$ resonant cavity LED driven by an arbitrary waveform generator (AWG, 81150A). The output signal from the C11209-110 was captured using a ZS1500 active probe and a HDO6014-MS oscilloscope. After synchronisation, the summation photon counting method was applied to the each bit period to determine the number of photons detected within a bit period and hence the value of the bit. In order to allow a comparison with the previously measured performance of a receiver contained an AD1900-9-TO5i APD[8] the number of photons required to achieve a BER of $7.9 \times 10^{-4}$ which was then measured.

Fig. 3 shows the ratio of number of detected photons per bit required to achieve this BER to the number required to achieve the same BER in the presence of Poisson noise. It can be seen that the C11209-110 receiver requires twice the number of detected photons per bit as an ideal Poisson limited receiver at $10 \mathrm{Mbps}$. However, as the data rate is increased the nonlinearity in Fig. 2 means that this ratio increases slowly until $100 \mathrm{Mbps}$. At higher data rates the bit time is shorter than the output pulse width and the resulting ISI means that the required number of detected photons per bit increases rapidly.

The results in Fig. 3 show that the new receiver is most effective when operated at data rates of $100 \mathrm{Mbps}$ or lower. However, the finite width of the output pulses means that at $100 \mathrm{Mbps}$ the receiver requires 5.5 times more photons per bit than a system limited by Poisson noise. In contrast, a receiver that includes an AD1900-9-TO5i APD with a MAX3665 transimpedance amplifier and a bandwidth of $90 \mathrm{MHz}$ requires approximately 10 times more detected photons per bit than the C11209-110 receiver to achieve the same BER. Unfortunately, at $650 \mathrm{~nm}$ the PDE of the S12571-010C in the C11209110 is only approximately $6 \%$. This inefficiency means that the APD will require a lower photon flux to achieve the same BER and data rate at $650 \mathrm{~nm}$. However, the PDE of the S12571-010C is more than $10 \%$ around $450 \mathrm{~nm}$ and so at this wavelength a receiver incorporating a C11209-110 would be as sensitive as the APD-based receiver. Another way to improve the sensitivity of a receiver would be to use an array of larger SPADs. For example, an S12571-

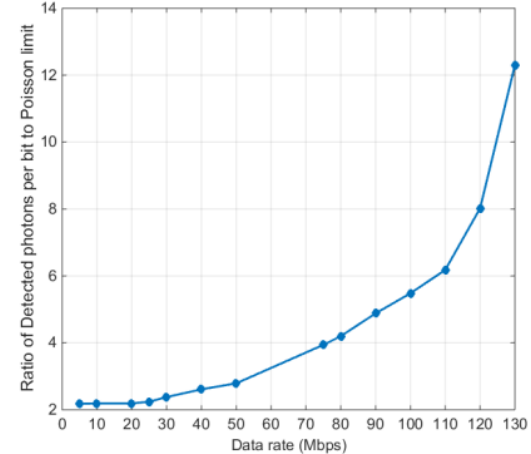

Fig. 3: The ratio of the detected photons per bit to the Poisson limit at different data rate to achieve a BER of $7.9 \times 10^{-4}$

$015 \mathrm{C}$ has a peak PDE of more than $25 \%$ and so a receiver containing this device will be 2.5 times more sensitive than the APD based receiver. Using even larger diameter SPADs, with a higher fill-factor and therefore PDE, could result in receivers that are ten times more sensitive than APD-based receivers.

\section{Conclusion}

Two simple methods of counting photons using a commercial off-the-shelf SPAD array have been described. Experimental results show that using one of these methods and wavelengths around $450 \mathrm{~nm}$ a SPAD-based receiver could be as sensitive as a receiver based upon an APD. However, it is anticipated that better photon counting methods and arrays of larger diameter SPADs will make possible SPAD-based receivers that are significantly more sensitive than any other receivers.

\section{References}

[1] H. Haas, L. Yin, S. Member, Y. Wang, S. Member, and I. Paper, "What is LiFi ?," J. Light. Technol ,vol. 34, no. 6, pp. 1533-1544, 2016.

[2] D. Chitnis and S. Collins, "A SPAD-based photon detecting system for optical communications," J. Light. Technol., vol. 32, no. 10, pp. 2028-2034, 2014.

[3] E. Fisher, I. Underwood, and R. Henderson, "A reconfigurable single-photon-counting integrating receiver for optical communications," IEEE J. Solid-State Circuits, vol. 48, no. 7, pp. 1638-1650, 2013.

[4] D. Chitnis, L. Zhang, H. Chun, S. Rajbhandari, G. Faulkner, D. O.Brien, and S. Collins, "A $200 \mathrm{Mb} / \mathrm{s}$ VLC demonstration with a SPAD based receiver," in IEEE Photonic summer topical meeting,2015,vol.3,pp. 226-227

[5] S. Gnecchi, N. A. W. Dutton, L. Parmesan, B. R. Rae, S. Pellegrini, S. J. Mcleod, L. A. Grant, and R. K. Henderson, "Analysis of Photon Detection Efficiency and Dynamic Range in SPAD-Based Visible Light Receivers," J. Light. Technol, vol. 34, no. 11, pp. 2774-2781, 2016.

[6] O. Almer, D. Tsonev, N. A. W. Dutton, T. Al Abbas, S. Videv, S.Gnecchi, H. Haas, and R. K. Henderson, "A SPAD-based Visible Light Communications Receiver Employing Higher OrderModulation," IEEE Global Telecommun. Conf, 2015.

[7] Kosman J. et al. $60 \mathrm{Mb} / \mathrm{s}, 2$ meters visible light communications in $1 \mathrm{klx}$ ambient using an unlensed CMOS SPAD receiver. IEEE Photonics Society Summer Topical Meeting Series (SUM), CA, USA

[8] L. Zhang, D. Chitnis, H. Chun, S. Rajbhandari, G. Faulkner, D. O.Brien, and S. Collins, "A comparision of APD and SPAD based receivers for Visible light communications" submitted to JSAC. 